Arch. Math. 81 (2003) 26-37

0003-889X/03/010026-12

DOI 10.1007/s00013-003-0512-y

(C) Birkhäuser Verlag, Basel, 2003

Archiv der Mathematik

\title{
Positive bilinear operators in Calderón-Lozanovskiĭ spaces
}

\author{
By
}

LECH MALIGRANDA

\begin{abstract}
A generalization of an abstract Hölder-Rogers inequality for positive bilinear operators is proved. Then it is used in the theory of interpolation of operators. An interpolation theorem for positive bilinear operators between Calderón-Lozanovskiı̌ spaces holds if and only if the parameter functions generating those spaces satisfy a generalized C-supermultiplicativity condition (2). In the case when all generating functions are the same this condition is exactly the same as the C-supermultiplicativity condition on the function.
\end{abstract}

0. Introduction. For a positive linear operator $T$ on $L^{0}(\mu)$ it is well-known that an abstract Hölder-Rogers inequality

$$
T(|x||y|) \leqq\left[T\left(|x|^{p}\right)\right]^{1 / p}\left[T\left(|y|^{q}\right)\right]^{1 / q},
$$

holds for any $x, y \in L^{0}(\mu)$, where $1 / p+1 / q=1, p>1$, and it can be generalized to the "function" version

$$
T(\varphi(|x|,|y|)) \leqq \varphi(T(|x|), T(|y|)) .
$$

We will extend the last inequality to positive bilinear operators and use it in the proof of bilinear interpolation result.

There are well known bilinear interpolation results for the real method of interpolation and also for the complex method even for general Banach couples (cf. [5]). For the CalderónLozanovskiı̆ spaces interpolation results for arbitrary linear (not only positive) operators are also known but for positive bilinear operators there is only a recent result of Astashkin [3] with some restrictions on the function generating the space and the Fatou property on the spaces in the image.

The next purpose of this paper is to find necessary and sufficient conditions on $\varphi$ under which we have interpolation results for positive bilinear operators in Calderón-Lozanovskiı̌ spaces. We will see that the additional assumptions on indices and the Fatou property on spaces, in the Astashkin result, are superfluous.

Mathematics Subject Classification (2000): 46E30, 46B42, 46B70.

Research supported by the Swedish Natural Science Research Council (NFR)-grant M5105-20005228/2000. 
The content of the paper is as follows. In Section 1 we define the Calderón-Lozanovskii spaces on the class of Banach function lattices.

Section 2 contains pointwise estimates for positive bilinear operators. Two proofs, each of independent interest, are presented there. The result obtained here is then applied to prove the interpolation property of different Calderón-Lozanovskiı̌ spaces for positive bilinear operators.

In Section 3 we prove that the Calderón-Lozanovskiĭ spaces generated by different functions have the interpolation property for positive bilinear operators if and only if these parameter functions satisfy a generalized C-supermultiplicativity condition (2). In the case when all generating functions are the same $\varphi_{0}=\varphi_{1}=\varphi$ this condition is exactly the same as the $\mathrm{C}$-supermultiplicativity condition on the function $\varphi$ and our result improve the recent theorem presented by Astashkin [3].

1. Calderón-Lozanovskii spaces. Let $(\Omega, \mu)$ be a complete $\sigma$-finite measure space and let $L^{0}(\mu)$ denote, as usual, the space of all equivalence classes of measurable functions on $\Omega$ with the topology of convergence in measure on $\mu$-finite sets. The order $|x| \leqq|y|$ means that $|x(t)| \leqq|y(t)|$ for $\mu$-almost all $t \in \Omega$.

If a normed subspace $E$ of $L^{0}(\mu)$ is such that there exists $u \in E$ with $u>0 \mu$-a.e. on $\Omega$ and $\|x\| \leqq\|y\|$ whenever $|x| \leqq|y|$, we say that $E$ is a normed function lattice (on $\Omega$ or on $(\Omega, \mu)$ ). A normed function lattice $E$ has the Fatou property if $0 \leqq x_{n} \uparrow x, x_{n} \in E$ and $\sup \left\|x_{n}\right\|_{E}<\infty$ implies $x \in E$ and $\left\|x_{n}\right\|_{E} \uparrow\|x\|_{E}$.

Recall that if $\bar{E}=\left(E_{0}, E_{1}\right)$ is a pair of Banach function lattices on $\Omega$ and $\mathcal{U}$ denotes the set of all non-negative concave and positively homogeneous continuous functions $\varphi$ : $[0, \infty) \times[0, \infty) \rightarrow[0, \infty)$ such that $\varphi(0,0)=0$. Then the Calderón-Lozanovskiu space $\varphi(\bar{E})=\varphi\left(E_{0}, E_{1}\right)$ consists of all $x \in L^{0}(\mu)$ such that $|x| \leqq \lambda \varphi\left(\left|x_{0}\right|,\left|x_{1}\right|\right)$ for some $x_{i} \in E_{i}$, with $\left\|x_{i}\right\|_{E_{i}} \leqq 1, i=0,1$. The spaces $\varphi(\bar{E})$ are Banach function lattices equipped with the norms

$$
\|x\|_{\varphi}=\inf \left\{\lambda>0 ;|x| \leqq \lambda \varphi\left(\left|x_{0}\right|,\left|x_{1}\right|\right),\left\|x_{0}\right\|_{E_{0}} \leqq 1,\left\|x_{1}\right\|_{E_{1}} \leqq 1\right\}
$$

(see [13]). In the case of the power functions $\varphi_{\theta}(s, t)=s^{1-\theta} t^{\theta}$ with $0 \leqq \theta \leqq 1, \varphi_{\theta}(\bar{E})$ are the well known Calderón spaces $E_{0}^{1-\theta} E_{1}^{\theta}$ (see [7]). Moreover, the particular case of $E^{1 / p}\left(L^{\infty}\right)^{1-1 / p}=E^{(p)}$ for $1<p<\infty$ is known as the p-convexification of $E$ (see [12]).

The properties of $\varphi(\bar{E})$ were studied by Lozanovskil in [13] and [14] (see also [16]), where among other facts it is proved the Köthe duality result

$$
\varphi\left(E_{0}, E_{1}\right)^{\prime}=\hat{\varphi}\left(E_{0}^{\prime}, E_{1}^{\prime}\right)
$$

with equivalent norms. Here, for $\varphi \in \mathcal{U}$, the conjugate function $\hat{\varphi}$ is defined by

$$
\hat{\varphi}(s, t):=\inf \left\{\frac{\alpha s+\beta t}{\varphi(\alpha, \beta)} ; \alpha, \beta>0\right\}, s, t \geqq 0 .
$$

We have $\hat{\varphi} \in \mathcal{U}$ and $\hat{\hat{\varphi}}=\varphi$ (see [14], Lemma 2, [15], Lemma 2 and [16], Lemma 15.8). 
Observe also that if $M:[0, \infty) \rightarrow[0, \infty]$ is a nondecreasing, convex and left-continuous function not identical 0 or $\infty$ on $(0, \infty)$ with $M(0)=0$, and $\varphi \in \mathcal{U}$ is defined by $\varphi(s, t)=$ $t M^{-1}(s / t)$ if $t>0$ and 0 if $t=0$, where $M^{-1}$ is the right continuous inverse of $M$, then, for any Banach lattice $E$ on $\Omega$, the Calderón-Lozanovskiu space $\varphi\left(E, L_{\infty}\right)$ coincides isometrically with the Banach lattice

$$
E_{M}=\left\{x \in L^{0}(\mu) ; M(|x| / \lambda) \in E \text { for some } \lambda>0\right\}
$$

equipped with the norm

$$
\|x\|_{E_{M}}=\inf \left\{\lambda>0 ;\|M(|x| / \lambda)\|_{E} \leqq 1\right\} .
$$

In particular, $\varphi\left(L_{1}, L_{\infty}\right)$ coincides isometrically with the Orlicz space $L_{M}$ (see [6], [16], [19]).

The subset of functions in $\mathcal{U}$ for which $\varphi(s, 1) \rightarrow 0$ as $s \rightarrow 0^{+}$and $\varphi(1, t) \rightarrow 0$ as $t \rightarrow 0^{+}$is denoted by $\mathcal{U}_{0}$. The subset of functions in $\mathcal{U}_{0}$ for which $\bar{\varphi}(s, 1) \rightarrow 0$ as $s \rightarrow 0^{+}$ and $\bar{\varphi}(1, t) \rightarrow 0$ as $t \rightarrow 0^{+}$, where $\bar{\varphi}(s, t)=\sup _{u, v>0} \frac{\varphi(s u, t v)}{\varphi(u, v)}$ is denoted by $\mathcal{U}^{ \pm}$.

Note that $\varphi \in \mathcal{U}^{ \pm}$if and only if the dilation indices (Orlicz-Matuszewska indices) of $\rho=\varphi(1, \cdot)$ are strictly between 0 and 1, i. e., $0<\alpha_{\rho} \leqq \beta_{\rho}<1$ (cf. [8], [16]).

2. Pointwise estimates for positive bilinear operators. Let us start with the result for positive linear operators which was used, independently by Berezhnoi (1981), Shestakov (1982) and Maligranda (1985), in the proof that the Calderón-Lozanovski1 spaces are exact interpolation spaces for positive linear operators (see [4], [21], [15]; see also [16], Th. 15.13). Since the result below was used but not written explicitely we include here a proof for the sake of completeness.

Lemma 1. Let $E$ be either $L^{0}\left(\mu_{1}\right)$ or a normed function lattice on $\left(\Omega_{1}, \mu_{1}\right)$ and let $T: E \rightarrow L^{0}(\mu)$ be a positive linear operator. If $\varphi \in \mathcal{U}$, then

$$
T(\varphi(|x|,|y|)) \leqq \varphi(T(|x|), T(|y|))
$$

for any $x, y \in E$.

Proof. Since

$$
\varphi(|x|,|y|) \leqq \frac{a|x|+b|y|}{\hat{\varphi}(a, b)} \quad(a>0, \quad b>0)
$$

it follows that

$$
T(\varphi(|x|,|y|)) \leqq \frac{a T(|x|)+b T(|y|)}{\hat{\varphi}(a, b)}
$$

for arbitrary $a, b>0$ and so by the fact that operation " $\leadsto$ " is an involution on $\mathcal{U}$

$$
T(\varphi(|x|,|y|)) \leqq \hat{\hat{\varphi}}(T(|x|), T(|y|))=\varphi(T(|x|), T(|y|)) .
$$


Remark 1 . As an easy consequence of Lemma 1 , for $\varphi(u, v)=u^{1 / p} v^{1-1 / p}$ with $1<p<\infty$, we obtain the inequality

$$
T\left(|x|^{1 / p}|y|^{1-1 / p}\right) \leqq[T(|x|)]^{1 / p}[T(|y|)]^{1-1 / p} .
$$

We can rewrite this as

$$
|T(x y)| \leqq T(|x||y|) \leqq\left[T\left(|x|^{p}\right)\right]^{1 / p}\left[T\left(|y|^{q}\right)\right]^{1 / q},
$$

where $1 / p+1 / q=1$. The last estimate is an abstract Hölder-Rogers inequality for positive linear operators, which is known in interpolation theory (see [8], p. 246) and in ergodic theory (see [9], Lemma 7.4).

Remark 2. Lemma 1 is true also for some nonlinear operators and functionals. An operator $T: E \rightarrow L^{0}(\mu)$ is called a positive sublinear operator if it is monotone, subadditive positively homogeneous, i. e., for any $x, y \in E$

$$
\begin{aligned}
& |x| \leqq y \Rightarrow|T(x)| \leqq T(y),|T(x+y)| \leqq|T(x)| \\
& \quad+|T(y)| \text { and }|T(\lambda x)| \leqq \lambda|T(x)| .
\end{aligned}
$$

We can easily see that for such operator $T$ the estimate (1) is still true. Similarly, for the positive sublinear functional $T$ on $E$ (the definition is analogous) estimate of type (1) is again true. In particular, by taking $T x=\|x\|_{E}$ we obtain that if $x, y \in E$, then $\varphi(|x|,|y|) \in E$ and

$$
\|\varphi(|x|,|y|)\|_{E} \leqq \varphi\left(\|x\|_{E},\|y\|_{E}\right)
$$

(see [15], Lemma 3(a), [16], Th. 15.9(a) and [11], p. 203; cf. also [20]).

Re mark 3. Lemma 1 can be written also in the following form: Let $E_{0}, E_{1}$ and $F_{0}, F_{1}$ be normed function lattices on $\left(\Omega_{1}, \mu_{1}\right)$ and $(\Omega, \mu)$, respectively. If $T: E_{0}+E_{1} \rightarrow F_{0}+F_{1}$ is a positive linear operator, then estimate (1) holds for any $x \in E_{0}, y \in E_{1}$.

Before we extend Lemma 1 to the bilinear case we prove some properties of functions $\varphi \in \mathcal{U}$.

Lemma 2. If $\varphi \in \mathcal{U}$, then

(i) $\varphi\left(s_{2}, t\right)-\varphi\left(s_{1}, t\right) \leqq \varphi\left(s_{2}-s_{1}, t\right)-\varphi(0, t)$ for all $s_{2}>s_{1}>0$ and $t>0$, where $\varphi(0, t)=\lim _{s \rightarrow 0^{+}} \varphi(s, t)$.

(ii) $\varphi\left(s, t_{2}\right)-\varphi\left(s, t_{1}\right) \leqq \varphi\left(s, t_{2}-t_{1}\right)-\varphi(s, 0)$ for all $t_{2}>t_{1}>0$ and $s>0$, where $\varphi(s, 0)=\lim _{t \rightarrow 0^{+}} \varphi(s, t)$.

Proof. (i). If $\varphi \in \mathcal{U}_{0}$, then

$$
\varphi(0, t)=\lim _{s \rightarrow 0^{+}} \varphi(s, t)=t \lim _{s \rightarrow 0^{+}} \varphi(s / t, 1)=0
$$


and the estimate follows directly from the subadditivity of $\varphi$ in the first variable because the function $\varphi$ is concave.

Assume now that $\varphi \in \mathcal{U} \backslash \mathcal{U}_{0}$. Put

$$
\varphi_{0}(s, t)=\varphi(s, t)-a_{\varphi} s-b_{\varphi} t,
$$

where

$$
a_{\varphi}=\lim _{t \rightarrow 0^{+}} \varphi(1, t) \text { and } b_{\varphi}=\lim _{s \rightarrow 0^{+}} \varphi(s, 1) .
$$

Then $\varphi \in \mathcal{U}_{0}$. The important here is to prove that $\varphi(s, t) \geqq a_{\varphi} s+b_{\varphi} t$ for all $s, t>0$. In fact, for $0<u<v<w$ we have by the concavity of $\varphi$ that

$$
\frac{\varphi(1, w)-\varphi(1, v)}{w-v} \leqq \frac{\varphi(1, v)-\varphi(1, u)}{v-u} .
$$

Taking $u \rightarrow 0^{+}$we obtain

$$
\frac{\varphi(1, w)-\varphi(1, v)}{w-v} \leqq \frac{\varphi(1, v)-a_{\varphi}}{v}
$$

or

$$
\varphi\left(\frac{1}{w}, 1\right)-\varphi\left(\frac{1}{w}, \frac{v}{w}\right) \leqq \frac{w-v}{w} \frac{\varphi(1, v)-a_{\varphi}}{v} .
$$

Taking $w \rightarrow \infty$ we get

$$
b_{\varphi} \leqq \frac{\varphi(1, v)-a_{\varphi}}{v}
$$

and with $v=t / s$ we have

$$
\frac{t}{s} b_{\varphi} \leqq \frac{\varphi(1, v)-a_{\varphi}}{v}
$$

or

$$
s a_{\varphi}+t b_{\varphi} \leqq \varphi(s, t) .
$$

For $\varphi \in \mathcal{U}_{0}$, by the subadditivity of $\varphi_{0}$, we obtain

$$
\begin{aligned}
\varphi\left(s_{2}, t\right)-\varphi\left(s_{1}, t\right) & =\varphi_{0}\left(s_{2}, t\right)+a_{\varphi} s_{2}+b_{\varphi} t-\left[\varphi_{0}\left(s_{1}, t\right)+a_{\varphi} s_{1}+b_{\varphi} t\right] \\
& =\varphi_{0}\left(s_{2}, t\right)-\varphi_{0}\left(s_{1}, t\right)+a_{\varphi}\left(s_{2}-s_{1}\right) \\
& \leqq \varphi_{0}\left(s_{2}-s_{1}, t\right)+a_{\varphi}\left(s_{2}-s_{1}\right) \\
& =\varphi\left(s_{2}-s_{1}, t\right)-a_{\varphi}\left(s_{2}-s_{1}\right)-b_{\varphi} t+a_{\varphi}\left(s_{2}-s_{1}\right) \\
& =\varphi\left(s_{2}-s_{1}, t\right)-b_{\varphi} t \\
& =\varphi\left(s_{2}-s_{1}, t\right)-\varphi(0, t)
\end{aligned}
$$

and the proof of (i) is finished. The proof of (ii) is similar. 
Now we extend Lemma 1 to bilinear operators. We will give even two proofs of this interesting estimate.

Let $E$ be either $L^{0}\left(\mu_{1}\right)$ or a normed function lattice on $\left(\Omega_{1}, \mu_{1}\right)$ and let $F$ be either $L^{0}\left(\mu_{2}\right)$ or a normed function lattice on $\left(\Omega_{2}, \mu_{2}\right)$. A bilinear operator $T: E \times F \rightarrow L^{0}(\mu)$ is called positive if $T(x, y) \geqq 0$ whenever $0 \leqq x \in E$ and $0 \leqq y \in F$.

Theorem 1. Let $\varphi_{0}, \varphi_{1}, \varphi \in \mathcal{U}$. Assume that either $T: L^{0}\left(\mu_{1}\right) \times L^{0}\left(\mu_{2}\right) \rightarrow L^{0}(\mu)$ or $T:(E+F) \times(E+F) \rightarrow L^{0}(\mu)$ is a positive bilinear operator. If

$$
\varphi_{0}(1, s) \varphi_{1}(1, t) \leqq C \varphi(1, s t)
$$

for some positive constant $C>0$ and all $s, t>0$, then

$$
T\left(\varphi_{0}\left(\left|x_{0}\right|,\left|x_{1}\right|\right), \varphi_{1}\left(\left|y_{0}\right|,\left|y_{1}\right|\right)\right) \leqq C \varphi\left(T\left(\left|x_{0}\right|,\left|x_{1}\right|\right), T\left(\left|y_{0}\right|,\left|y_{1}\right|\right)\right)
$$

for any $x_{0}, y_{0} \in E$ and $x_{1}, y_{1} \in F$.

First pro of. We will use here Lemma 1 and Lemma 2. The idea of the first proof is coming from Shestakov [21].

It is enough to show (3) for any $0 \leqq x_{0}, y_{0} \in E$ and $0 \leqq x_{1}, y_{1} \in F$.

Let $y=y_{0}+y_{1}$. Suppose first that $y_{0}$ and $y_{1}$ are $y$-step functions, i. e.,

$$
y_{0}=\sum_{k=1}^{m} \alpha_{k} z_{k}, \quad y_{1}=\sum_{k=1}^{m} \beta_{k} z_{k},
$$

where $\alpha_{k}, \beta_{k} \geqq 0, k=1,2, \ldots, m, z_{1}, z_{2}, \ldots, z_{m}$ are pairwise disjoint with $\sum_{k=1}^{m} z_{k}=y$ (cf. [1], p. 81). For example, as $z_{k}$ we can take $z_{k}=y \chi_{A_{k}}$, where $A_{k}$ are measurable and pairwise disjoint subsets of $\Omega$ such that $\bigcup_{k=1}^{m} A_{k}=\Omega$.

Since $T$ is a positive homogeneous and additive in the second variable it follows that

$$
\begin{aligned}
T\left(\varphi_{0}\left(x_{0}, x_{1}\right), \varphi_{1}\left(y_{0}, y_{1}\right)\right) & =T\left(\varphi_{0}\left(x_{0}, x_{1}\right), \sum_{k=1}^{m} \varphi_{1}\left(\alpha_{k}, \beta_{k}\right) z_{k}\right) \\
& =\sum_{k=1}^{m} \varphi_{1}\left(\alpha_{k}, \beta_{k}\right) T\left(\varphi_{0}\left(x_{0}, x_{1}\right), z_{k}\right),
\end{aligned}
$$

and then by using Lemma 1 to the first variable with the second variable fixed, the assumption (2) and the concavity of $\varphi$ we find that

$$
\begin{aligned}
T\left(\varphi_{0}\left(x_{0}, x_{1}\right), \varphi_{1}\left(y_{0}, y_{1}\right)\right) & \leqq \sum_{k=1}^{m} \varphi_{1}\left(\alpha_{k}, \beta_{k}\right) \varphi_{0}\left(T\left(x_{0}, z_{k}\right), T\left(x_{1}, z_{k}\right)\right) \\
& \leqq C \sum_{k=1}^{m} \varphi\left(\alpha_{k} T\left(x_{0}, z_{k}\right), \beta_{k} T\left(x_{1}, z_{k}\right)\right)
\end{aligned}
$$




$$
\begin{aligned}
& \leqq C \varphi\left(\sum_{k=1}^{m} \alpha_{k} T\left(x_{0}, z_{k}\right), \sum_{k=1}^{m} \beta_{k} T\left(x_{1}, z_{k}\right)\right) \\
& =C \varphi\left(T\left(x_{0}, x_{1}\right), T\left(y_{0}, y_{1}\right)\right) .
\end{aligned}
$$

If $0 \leqq y_{i} \in Y_{i}, i=0,1$ are arbitrary, then we can find sequences $\left\{y_{n}^{0}\right\},\left\{y_{n}^{1}\right\}$ of y-step functions such that

$$
0 \leqq y_{n}^{i} \leqq y_{i}, \quad y_{i}-y_{n}^{i} \leqq \frac{y}{n} \text { for } n=1,2, \ldots, \quad \mathrm{i}=0,1 .
$$

Then, by Lemma 2 ,

$$
\begin{aligned}
0 & \leqq \varphi_{1}\left(y_{0}, y_{1}\right)-\varphi_{1}\left(y_{n}^{0}, y_{n}^{1}\right) \\
& =\varphi_{1}\left(y_{0}, y_{1}\right)-\varphi_{1}\left(y_{n}^{0}, y_{1}\right)+\varphi_{1}\left(y_{n}^{0}, y_{1}\right)-\varphi_{1}\left(y_{n}^{0}, y_{n}^{1}\right) \\
& \leqq \varphi_{1}\left(y_{0}-y_{n}^{0}, y_{1}\right)-\varphi_{1}\left(0, y_{1}\right)+\varphi_{1}\left(y_{n}^{0}, y_{1}-y_{n}^{1}\right)-\varphi_{1}\left(y_{0}, 0\right) \\
& \leqq \varphi_{1}\left(\frac{y}{n}, y_{1}\right)-\varphi_{1}\left(0, y_{1}\right)+\varphi_{1}\left(y_{n}^{0}, \frac{y}{n}\right)-\varphi_{1}\left(y_{0}, 0\right) \\
& \leqq \lambda_{n}\left(y_{0}+y_{1}\right),
\end{aligned}
$$

with

$$
\lambda_{n}=\varphi_{1}\left(\frac{1}{n}, 1\right)-\varphi_{1}(0,1)+\varphi_{1}\left(1, \frac{1}{n}\right)-\varphi_{1}(1,0) .
$$

Thus

$$
\varphi_{1}\left(y_{0}, y_{1}\right) \leqq \varphi_{1}\left(y_{n}^{0}, y_{n}^{1}\right)+\lambda_{n}\left(y_{0}+y_{1}\right),
$$

and by the first part of the proof and the chosen sequences

$$
\begin{aligned}
& T\left(\varphi_{0}\left(x_{0}, x_{1}\right), \varphi_{1}\left(y_{0}, y_{1}\right)\right) \leqq T\left(\varphi_{0}\left(x_{0}, x_{1}\right), \varphi_{1}\left(y_{n}^{0}, y_{n}^{1}\right)\right) \\
& \quad+\lambda_{n} T\left(\varphi_{0}\left(x_{0}, x_{1}\right), y_{0}+y_{1}\right) \\
& \quad \leqq C \varphi\left(T\left(x_{0}, x_{1}\right), T\left(y_{n}^{0}, y_{n}^{1}\right)\right)+\lambda_{n} T\left(\varphi_{0}\left(x_{0}, x_{1}\right), y_{0}+y_{1}\right) \\
& \quad \leqq C \varphi\left(T\left(x_{0}, x_{1}\right), T\left(y_{0}, y_{1}\right)\right)+\lambda_{n} T\left(\varphi_{0}\left(x_{0}, x_{1}\right), y_{0}+y_{1}\right) .
\end{aligned}
$$

Since by the continuity of the function $\varphi_{1}$ the sequence $\lambda_{n} \rightarrow 0$ as $n \rightarrow \infty$ it follows that

$$
T\left(\varphi_{0}\left(x_{0}, x_{1}\right), \varphi_{1}\left(y_{0}, y_{1}\right)\right) \leqq C \varphi\left(T\left(x_{0}, x_{1}\right), T\left(y_{0}, y_{1}\right)\right)
$$

and the proof is complete.

Sec ond proof. The idea of the second proof is similar to the one of Lemma 1. Let $0 \leqq x_{0}, y_{0} \in E$ and $0 \leqq x_{1}, y_{1} \in F$. Then, using the definition of $\hat{\varphi}_{1}$, the positivity and bilinearity of $T$, and Lemma 1 , we have for any $a, b>0$,

$$
\begin{aligned}
& T\left(\varphi_{0}\left(x_{0}, x_{1}\right), \varphi_{1}\left(y_{0}, y_{1}\right)\right) \leqq T\left(\varphi_{0}\left(x_{0}, x_{1}\right), \frac{a y_{0}+b y_{1}}{\hat{\varphi}_{1}(a, b)}\right) \\
& \quad=\frac{a}{\hat{\varphi}_{1}(a, b)} T\left(\varphi_{0}\left(x_{0}, x_{1}\right), y_{0}\right)+\frac{b}{\hat{\varphi}_{1}(a, b)} T\left(\varphi_{0}\left(x_{0}, x_{1}\right), y_{1}\right)
\end{aligned}
$$




$$
\begin{aligned}
\leqq & \frac{a}{\hat{\varphi}_{1}(a, b)} \varphi_{0}\left(T\left(x_{0}, y_{0}\right), T\left(x_{1}, y_{0}\right)\right) \\
& +\frac{b}{\hat{\varphi}_{1}(a, b)} \varphi_{0}\left(T\left(x_{0}, y_{1}\right), T\left(x_{1}, y_{1}\right)\right)=A .
\end{aligned}
$$

Since

$$
\begin{aligned}
\frac{\varphi_{0}(u, v)}{\hat{\varphi}_{1}(a, b)} & =\sup _{s, t>0} \frac{\varphi_{0}(u, v) \varphi_{1}(s, t)}{a s+b t} \leqq C \sup _{s, t>0} \frac{\varphi(s u, t v)}{s a+t b} \\
& =\frac{C}{\inf _{s, t>0} \frac{s a+t b}{\varphi(s u, t v)}}=\frac{C}{\hat{\varphi}(a / u, b / v)}
\end{aligned}
$$

it follows that

$$
\begin{aligned}
A & \leqq C\left[\frac{a}{\hat{\varphi}\left(a / T\left(x_{0}, y_{0}\right), b / T\left(x_{1}, y_{0}\right)\right)}+\frac{b}{\hat{\varphi}\left(a / T\left(x_{0}, y_{1}\right), b / T\left(x_{1}, y_{1}\right)\right)}\right] \\
& \leqq C\left[\frac{a_{0} T\left(x_{0}, y_{0}\right)+b_{0} \epsilon_{0}}{\hat{\varphi}\left(a_{0}, b_{0}\right)}+\frac{b_{1} T\left(x_{1}, y_{1}\right)+a_{1} \epsilon_{1}}{\hat{\varphi}\left(a_{1}, b_{1}\right)}\right],
\end{aligned}
$$

where $a_{i}=a / T\left(x_{0}, y_{i}\right), b_{i}=b / T\left(x_{1}, y_{i}\right), i=0,1$ and $\epsilon_{0}, \epsilon_{1}>0$ are arbitrary.

Taking the infimum over all $a_{0}, b_{0}, a_{1}, b_{1}>0$ and using the facts that the operation " :" $^{\prime \prime}$ is an involution on $\mathcal{U}$ and $\varphi$ is a concave function we obtain

$$
\begin{aligned}
A & \leqq C\left[\hat{\hat{\varphi}}\left(T\left(x_{0}, y_{0}\right), \epsilon_{0}\right)+\hat{\hat{\varphi}}\left(\epsilon_{1}, T\left(x_{1}, y_{1}\right)\right)\right] \\
& =C\left[\varphi\left(T\left(x_{0}, y_{0}\right), \epsilon_{0}\right)+\varphi\left(\epsilon_{1}, T\left(x_{1}, y_{1}\right)\right)\right] \\
& \leqq C \varphi\left(T\left(x_{0}, y_{0}\right)+\epsilon_{0}, T\left(x_{1}, y_{1}\right)+\epsilon_{1}\right) .
\end{aligned}
$$

Since $\varphi$ is continuous and $\epsilon_{0}, \epsilon_{1}>0$ were arbitrary we get the estimate (3) and the proof is again finished.

Re m ark 4. Theorem 1 can be also proved for positive multilinear ( $n$-linear) operators with the following assumption on the functions in $\mathcal{U}$ :

$$
\varphi_{0}\left(1, s_{0}\right) \varphi_{1}\left(1, s_{1}\right) \cdots \varphi_{n-1}\left(1, s_{n-1}\right) \leqq C \varphi\left(1, s_{0} s_{1} \cdots s_{n-1}\right)
$$

for some $C>0$ and all $s_{0}, s_{1}, \ldots, s_{n-1}>0$.

\section{Interpolation of positive bilinear operators on Calderón-Lozanovskii spaces.}

As we have already mentioned before the Calderón-Lozanovskiľ spaces are exact interpolation spaces for positive linear operators (see Berezhnoi [4], Shestakov [21], Maligranda [15]; cf. also [16], Th. 15.13). Morover,

$$
\|T\|_{\varphi(\bar{E}) \rightarrow \varphi(\bar{F})} \leqq \bar{\varphi}\left(\|T\|_{E_{0} \rightarrow F_{0}},\|T\|_{E_{1} \rightarrow F_{1}}\right),
$$

for any $T \in L\left(\left\{E_{0}, E_{1}\right\},\left\{F_{0}, F_{1}\right\}\right)$. 
For arbitrary linear operators (not necessary positive) the history of interpolation results is the following (cf. [8], [10], [16]): Lozanovskii proved in 1972 that $\varphi(\cdot)$ is not an interpolation method even for $\varphi(s, t)=s^{1-\theta} t^{\theta}$. Moreover, Calderón (1964), Zabreiko (1967), Shestakov (1974) and Lozanovskii $(1976,1979)$ have been given some conditions under which $E_{0}^{1-\theta} E_{1}^{\theta}$ is an interpolation method. For the case $\varphi\left(E_{0}, E_{1}\right)$ these results were proved by Ovchinnikov (1976, 1984), Berezhnoi (1980), Shestakov (1981), Maligranda (1985, 1989), Brudnyı̆-Krugljak (1991), Kruglyak-Maligranda-Persson (1993), and moreover for the class of quasi-Banach function lattices by Ovchinnikov (1982) and Nilsson (1985).

Astashkin [3] started in 1997 with a result for positive bilinear operators between the Calderón-Lozanovskiǐ spaces generated by some function $\varphi \in \mathcal{U}^{ \pm}$and the Fatou property of the spaces in the image. In fact, he announced the bilinear case at the conference in 1995 (cf. [2], Th. 2). His method of the proof was based on a calculation of the positive orbit. We generalize his result to any $\varphi \in \mathcal{U}$ (even to three functions $\varphi_{0}, \varphi_{1}, \varphi \in \mathcal{U}$ ) and to general Banach function lattices. Our proof here is different.

Theorem 2. Let $\bar{E}=\left(E_{0}, E_{1}\right), \bar{F}=\left(F_{0}, F_{1}\right), \bar{G}=\left(G_{0}, G_{1}\right)$ be couples of Banach function lattices on the measure spaces $\left(\Omega_{1}, \mu_{1}\right),\left(\Omega_{2}, \mu_{2}\right)$ and $(\Omega, \mu)$, respectively. Assume that $T:\left(E_{0}+E_{1}\right) \times\left(F_{0}+F_{1}\right) \rightarrow G_{0}+G_{1}$ is a positive bilinear operator such that the restriction $T: E_{i} \times F_{i} \rightarrow G_{i}$ is bounded for $i=0,1$. If $\varphi_{0}, \varphi_{1}, \varphi \in \mathcal{U}$ are such that

$$
\varphi_{0}(1, s) \varphi_{1}(1, t) \leqq C \varphi(1, s t)
$$

for some positive constant $C>0$ and all $s, t>0$, then

$$
T: \varphi_{0}(\bar{E}) \times \varphi_{1}(\bar{F}) \rightarrow \varphi(\bar{G})
$$

is bounded and

$$
\|T\|_{\varphi(\bar{E}) \times \varphi(\bar{F}) \rightarrow \varphi(\bar{G})} \leqq C \bar{\varphi}\left(\|T\|_{E_{0} \times F_{0} \rightarrow G_{0}},\|T\|_{E_{1} \times F_{1} \rightarrow G_{1}}\right) .
$$

The result is sharp in the sense that if (7) holds for any Banach couples of function lattices $\bar{E}, \bar{F}, \bar{G}$, then condition (5) on functions must be satisfied.

Proof. First, let us observe that Theorem 1 can be also written in the following form: for the above operator $T$ with the assumption (5) on the functions $\varphi_{0}, \varphi_{1}, \varphi$ we have that the estimate (3) holds for any $x_{i} \in E_{i}, y_{i} \in F_{i}, i=0,1$.

Now, let $x \in \varphi_{0}(\bar{E}), y \in \varphi_{1}(\bar{F})$ and $\|x\|_{\varphi_{0}(\bar{E})}<1,\|y\|_{\varphi_{1}(\bar{F})}<1$. Then there exist $x_{i} \in E_{i}, y_{i} \in F_{i}$ with $\left\|x_{i}\right\|_{E_{i}} \leqq 1,\left\|y_{i}\right\|_{F_{i}} \leqq 1$ for $i=0,1$ such that

$$
|x| \leqq \varphi_{0}\left(\left|x_{0}\right|,\left|x_{1}\right|\right) \text { and }|y| \leqq \varphi_{1}\left(\left|y_{0}\right|,\left|y_{1}\right|\right) \text {. }
$$

In view of the monotonicity of $T$ and by using Theorem 1 (in fact, our first observation at the beginning of the proof here) we obtain

$$
\begin{aligned}
|T(x, y)| & \leqq T(|x|,|y|) \\
& \leqq T\left(\varphi_{0}\left(\left|x_{0}\right|,\left|x_{1}\right|\right), \varphi_{1}\left(\left|y_{0}\right|,\left|y_{1}\right|\right)\right) \\
& \leqq C \varphi\left(T\left(\left|x_{0}\right|,\left|x_{1}\right|\right), T\left(\left|y_{0}\right|,\left|y_{1}\right|\right)\right) .
\end{aligned}
$$


Denote $M_{i}=\|T\|_{E_{i} \times F_{i} \rightarrow G_{i}}, i=0,1$, and $z_{0}=\left(T\left(\left|x_{0}\right|,\left|x_{1}\right|\right) / M_{0}, z_{1}=T\left(\left|y_{0}\right|,\left|y_{1}\right|\right) / M_{1}\right.$. Then

$$
\begin{aligned}
|T(x, y)| & \leqq C \varphi\left(T\left(\left|x_{0}\right|,\left|x_{1}\right|\right), T\left(\left|y_{0}\right|,\left|y_{1}\right|\right)\right) \\
& =C \varphi\left(M_{0} z_{0}, M_{1} z_{1}\right) \\
& \leqq C \bar{\varphi}\left(M_{0}, M_{1}\right) \varphi\left(z_{0}, z_{1}\right) .
\end{aligned}
$$

Since $\left\|z_{i}\right\|_{G_{i}} \leqq 1, i=0,1$ it follows that

$$
T(x, y) \in \varphi(\bar{G}) \text { and }\|T(x, y)\|_{\varphi(\bar{G})} \leqq C \bar{\varphi}\left(M_{0}, M_{1}\right),
$$

from which we have the estimate (7).

The necessity of inequality (5) we show by considering concrete positive bilinear operator and concrete spaces.

Let $\bar{E}=\bar{F}=\left(L_{1}, L_{\infty}\right)$ on the measure space $\left(R_{+}, m_{1}\right)$ and $\bar{G}=\left(L_{1}, L_{\infty}\right)$ on the measure space $\left(R_{+}^{2}, m_{2}\right)$, where $m_{1}$ and $m_{2}$ are Lebesgue measures on $R_{+}$and $R_{+}^{2}$, respectively.

Consider the tensor product map $T:\left(L_{1}+L_{\infty}\right) \times\left(L_{1}+L_{\infty}\right) \rightarrow L_{1}+L_{\infty}$ defined by

$$
T(x, y)(u, v)=x(u) y(v), u, v \in R_{+} .
$$

Then $T$ is a positive bilinear operator with the norms

$$
\|T\|_{L_{i} \times L_{i} \rightarrow L_{i}}=1, i=1, \infty .
$$

Assume that (6) holds. Then

$$
\|T(x, y)\|_{\varphi\left(L_{1}, L_{\infty}\right)} \leqq C\|x\|_{\varphi_{0}\left(L_{1}, L_{\infty}\right)}\|y\|_{\varphi_{1}\left(L_{1}, L_{\infty}\right)}
$$

for any $x \in \varphi_{0}\left(L_{1}, L_{\infty}\right), y \in \varphi_{1}\left(L_{1}, L_{\infty}\right)$.

Taking now $x=\chi_{(0, s)}, y=\chi_{(0, t)}, s, t>0$ and using the facts that

$$
\left\|\chi_{(0, r)}\right\|_{\varphi_{i}\left(L_{1}, L_{\infty}\right)}=\left\|\chi_{(0, r)}\right\|_{L_{M_{i}}}=\frac{1}{M_{i}^{-1}(1 / r)}=\varphi_{i}(1, r), \quad i=0,1,
$$

and

$$
\left\|\chi_{(0, s)} \chi_{(0, t)}\right\|_{L_{M}\left(m_{2}\right)}=\frac{1}{M^{-1}(1 /(s t))}=\varphi(1, s t)
$$

we obtain

$$
\varphi(1, s t) \leqq C \varphi_{0}(1, s) \varphi_{1}(1, t)
$$

for all $s, t>0$. The proof is complete.

Remark 5. Theorem 2 is still true for some nonlinear operators, namely for positive sublinear operators in each variable. Moreover, if $T:\left(E_{0}+E_{1}\right) \times\left(F_{0}+F_{1}\right) \rightarrow G_{0}+G_{1}$ is a bilinear operator dominated by a positive bilinear $S$ such that $S: E_{i} \times F_{i} \rightarrow G_{i}$ is bounded for $i=0,1$, then

$$
\|T\|_{\varphi(\bar{E}) \times \varphi(\bar{F}) \rightarrow \varphi(\bar{G})} \leqq C \bar{\varphi}\left(\|S\|_{E_{0} \times F_{0} \rightarrow G_{0}},\|S\|_{E_{1} \times F_{1} \rightarrow G_{1}}\right) .
$$


Remark 6. Theorem 2 can be easily formulated for positive multilinear ( $n$-linear) operators and the corresponding assumption to (5) will be

$$
\varphi_{0}\left(1, s_{0}\right) \varphi_{1}\left(1, s_{1}\right) \cdots \varphi_{n-1}\left(1, s_{n-1}\right) \leqq C \varphi\left(1, s_{0} s_{1} \cdots s_{n-1}\right)
$$

for some $C>0$ and all $s_{0}, s_{1}, \ldots, s_{n-1}>0$.

Corollary 3. Let $\bar{E}=\left(E_{0}, E_{1}\right), \bar{F}=\left(F_{0}, F_{1}\right), \bar{G}=\left(G_{0}, G_{1}\right)$ be couples of Banach function lattices on the measure spaces $\left(\Omega_{1}, \mu_{1}\right),\left(\Omega_{2}, \mu_{2}\right)$ and $(\Omega, \mu)$, respectively. Assume that $T:\left(E_{0}+E_{1}\right) \times\left(F_{0}+F_{1}\right) \rightarrow G_{0}+G_{1}$ is a positive bilinear operator such that the restriction $T: E_{i} \times F_{i} \rightarrow G_{i}$ is bounded for $i=0$, 1. If $\varphi \in \mathcal{U}$ is a $C$-supermultiplicative function, i. e.,

$$
\varphi(1, s) \varphi(1, t) \leqq C \varphi(1, s t)
$$

for some positive constant $C>0$ and all $s, t>0$, then

$$
T: \varphi(\bar{E}) \times \varphi(\bar{F}) \rightarrow \varphi(\bar{G})
$$

is bounded and

$$
\|T\|_{\varphi(\bar{E}) \times \varphi(\bar{F}) \rightarrow \varphi(\bar{G})} \leqq C \bar{\varphi}\left(\|T\|_{E_{0} \times F_{0} \rightarrow G_{0}},\|T\|_{E_{1} \times F_{1} \rightarrow G_{1}}\right) .
$$

The result is sharp in the sense that if (10) holds for any Banach couples of function lattices $\bar{E}, \bar{F}, \bar{G}$, then the function $\varphi$ is $C$-supermultiplicative for some $C>0$.

Re mark 7. Corollary 3 for the C-supermultiplicative function $\varphi$ and with additional assumptions that $\varphi \in \mathcal{U}^{ \pm}$and $G_{0}, G_{1}$ have the Fatou property was proved by Astashkin [3], Th. 1. He posed the question if the additional assumption $\varphi \in \mathcal{U}^{ \pm}$is necessary. We see, from Corollary 3 , that this additional assumption is superfluous which gives then an answer to his problem. Moreover, the Fatou property on $G_{0}, G_{1}$ is also not needed.

What is known about interpolation of arbitrary bilinear operators (not necessary positive) between Calderón-Lozanovskiǔ spaces? Of course, we need the C-supermultiplicativity of $\varphi$ but what else?

In the case of the power functions $\varphi_{\theta}(s, t)=s^{1-\theta} t^{\theta}$ with $0 \leqq \theta \leqq 1$ and on the class of Banach function lattices with continuous norms the spaces $\varphi_{\theta}(\overline{\bar{E}})$ are equal to the complex Calderón interpolation method $\left[E_{0}, E_{1}\right]_{\theta}$ for which the bilinear interpolation theorem holds (see [7],[5]).

We finally formulate two problems on bilinear interpolation.

Proble m 1 (cf. [16], Problem 10). When is $\varphi(\cdot, \cdot)$ an interpolation method for bilinear operators?

Proble m 2. Is Theorem 2, with the assumption (5) on the functions, true for arbitrary bilinear operators? 


\section{References}

[1] C. D. Aliprantis and O. Burkinshaw, Positive Operators, Orlando 1985.

[2] S. V. AstashKIN, The bilinear interpolation theorem for spaces of the Calderón-Lozanovskii method. In: Abstracts of the Internat. Conf. on "Function Spaces, Approximation Theory, Nonlinear Analysis" (April 27 - May 3, 1995). Moscow 319-320, 1995.

[3] S. V. ASTASHKIN, Interpolation of positive polylinear operators in Calderón-Lozanovskiǔ spaces. Sibirsk. Mat. Zh. 38, 1211-1218 (1997); English transl.: Siberian Math. J. 38, 1047-1053 (1997).

[4] E. I. BEREZHNOI, Interpolation of positive operators in the spaces $\varphi\left(X_{0}, X_{1}\right)$. In: Qualitative and Approximate Methods for the Investigation of Operator Equations. Yaroslav Gos. Univ. 3-12 Yaroslavl 1981 (Russian).

[5] J. BERGH and J. LÖFSTRÖM, Interpolation Spaces. Berlin 1976.

[6] Y. A. BRUDNYY and N. Y. KRUGLJAK, Interpolation Functors and Interpolation Spaces I. Amsterdam 1991.

[7] A. P. CALDERÓN, Intermediate spaces and interpolation, the complex method. Studia Math. 24, 113-190 (1964).

[8] S. G. KRein, Y. I. Petunin and E. M. SemEnOv, Interpolation of Linear Operators. Nauka, Moscow 1978; English transl.: Amer. Math. Soc., Providence 1982.

[9] U. KRENGEL, Ergodic Theorems. Berlin 1985.

[10] N. Y. Kruglyak, L. Maligranda and L. E. Persson, A Carlson type inequality with blocks and interpolation, Studia Math. 104, 161-180 (1993).

[11] M. KucZMA, An Introduction to the Theory of Functional Equations and Inequalities. Warsaw 1985.

[12] J. LindENSTRAusS and L. TZAFRIRI, Classical Banach Spaces, II. Function Spaces. Berlin-New York 1979.

[13] G. Y. LoZANOVSKII, On some Banach lattices IV. Sibirsk. Mat. Z. 14, 140-155 (1973). English transl.: Siberian. Math. J. 14, 97-108 (1973).

[14] G. Y. LOZANOVSKIr, Transformations of ideal Banach spaces by means of concave functions. In: Qualitative and Approximate Methods for the Investigation of Operator Equations. Yaroslav. Gos. Univ. 122-147 Yaroslavl 1978 (Russian).

[15] L. Maligranda, Calderón-Lozanovskiĭ spaces and interpolation of operators. Semesterbericht Funktionalanal. Tübingen 8, 83-92 (1985).

[16] L. MALIGRANDA, Orlicz Spaces and Interpolation. Sem Math. 5, Univ. of Campinas, Campinas SP, Brazil 1989.

[17] P. NILSSON, Interpolation of Banach lattices. Studia Math. 82, 135-154 (1985).

[18] V. I. OVCHINNIKOV, Interpolation theorems resulting from Grothendieck's inequality. Funkcional. Anal. i Priložhen. 10, no. 4, 45-54 (1976); English transl.: Functional Anal. Appl. 10, 287-294 (1977).

[19] V. I. OvchinNikov, The Methods of Orbits in Interpolation Theory. Math. Rep. Vol.1, Part 2, 349-516 1984

[20] J. E. PeČARIĆ and D. VelJan, On Maligranda's generalization of Jensen's inequality, J. Math. Anal. Appl. 200, 121-125 (1996).

[21] V. A. SHESTAKOV, Transformations of Banach ideal spaces and interpolation of linear operators. Bull. Polon. Acad. Sci. Math. 29, 569-577 (1981) (Russian).

Received: 7 December 2001; revised manuscript accepted: 16 September 2002

Lech Maligranda

Department of Mathematics

Luleå University of Technology

SE-971 87 Luleå

Sweden

lech@sm.luth.se 